\title{
Meropenem and piperacillin-tazobactam levels for critical care patients during empiric therapy
}

\section{Dear Editor,}

Individualised beta-lactam dosing in critically ill patients has been proposed as these patients have substantially altered pharmacokinetics (PK) of beta-lactams due to hypoalbuminaemia, aggressive fluid resuscitation, the existence of organ failure(s), as well as augmented renal clearance..$^{1-4}$ These physiologic changes are the basis for the significant inter-patient PK variability observed among the critically ill. In addition, intra-individual variability must also be anticipated due to rapidly changing clinical condition towards either improvement and cure, or deterioration and organ failures, after as short as 4 days of treatment. ${ }^{5}$ Given that antibiotic dosing regimens are derived from healthy volunteers or non-critically ill patients and do not account for such PK variations, standard antibiotic dosing regimens may not be appropriate for the critically ill.

Pharmacodynamic effect of beta-lactams is described by the free drug concentrations above bacterial minimum inhibitory concentrations (MIC) over a desired percentage of the dosing period $(\% f \mathrm{~T}>\mathrm{MIC})$. These time-dependent antibiotics achieve more bacterial killing the longer they remain at serum levels above the MIC. ${ }^{6}$ In vitro and in vivo animal studies have demonstrated that beta-lactam concentration should be maintained above the MIC between $40 \%$ and $70 \%$ of the dosing interval. ${ }^{7}$ Improved clinical and microbiological outcomes have been demonstrated in patients with at least $100 \% f \mathrm{~T}>\mathrm{MIC}$ for beta-lactams, especially in the critically ill. ${ }^{2}$ A study also reported that clinical cure in critically ill patients required betalactam plasma concentration reaching 4 to 6 times the MIC to ensure adequate tissue penetration and to prevent resistance development. ${ }^{6}$

Given the lack of data on critically ill Asian patients, we aim to determine total meropenem and piperacillin component of piperacillin-tazobactam concentration in the first 24-48 hours (pre-steady state) and at day 3-4 (steady state) in our population.

A prospective observational study was carried out from July 2016 to March 2017 in the medical and surgical intensive care units (ICUs) of Tan Tock Seng Hospital, a 1,700-bed acute-care hospital in Singapore. Ethics approval and informed consent from patients or legal representatives were obtained. The inclusion criteria were adult patients ( $\geq 21$ years), ICU admission and administration of piperacillin-tazobactam or meropenem. The exclusion criteria were expected mortality within 48 hours and pregnancy.

During pre-steady state, blood was drawn at 30 minutes, 1.5 hours and 3 hours from the start of infusion, and 30 minutes before the next dose. This was repeated at steady state. All assays were performed using liquid chromatography-tandem mass spectrometry. PK modelling and Monte Carlo simulations were performed using non-parametric adaptive grid algorithm in Pmetrics version 1.5.0 (Laboratory of Applied Pharmacokinetics and Bioinformatics, Los Angeles, US) using a onecompartment model. Free drug concentration was estimated based on published protein binding values ( $2 \%$ for meropenem and $30 \%$ for piperacillin).

Among the total of 42 patients, 16 (38.1\%) were prescribed piperacillin-tazobactam and $26(61.9 \%)$ were prescribed meropenem. Patients were predominantly male $(31 / 42,73.8 \%)$. Median age was 70 years (interquartile range, IQR 60-74), median total body weight was $60 \mathrm{~kg}$ (IQR 55-70) and median Acute Physiologic Assessment and Chronic Health Evaluation II (APACHE II) score was 23 (IQR 20-28) on day 1 of blood taking. Majority $(34 / 42,81.0 \%)$ required mechanical ventilation and inotropic support. Median calculated creatinine clearance using the CockcroftGault formula was $29 \mathrm{~mL} / \mathrm{min}$ (IQR $17-55 \mathrm{~mL} / \mathrm{min}$ ), and 9 patients $(21.4 \%)$ required dialysis. The most common sources of infections were pneumonia $(30 / 42,71.4 \%)$, unspecified sepsis $(8 / 42,19.0 \%)$ and bacteremia $(4 / 42,9.5 \%)$. All patients received doses according to manufacturers' product information leaflet. Only 8 $(19.0 \%)$ received extended infusion. Enterobacteriaceae spp. was most frequently isolated $(12 / 18,66.7 \%)$. Among these, the non-susceptible organisms had meropenem MIC of $4 \mathrm{mg} / \mathrm{L}$ (1 isolate); piperacillin MIC of $32 \mathrm{mg} / \mathrm{L}$ ( 1 isolate) and $\geq 128 \mathrm{mg} / \mathrm{L}$ (1 isolate); the remaining were susceptible (piperacillin-tazobactam, piperacillin $\mathrm{MIC} \leq 16 \mathrm{mg} / \mathrm{L}$, meropenem $\mathrm{MIC} \leq 0.25 \mathrm{mg} / \mathrm{L}$ ).

Attainment of PK targets for meropenem and piperacillin-tazobactam are reported in Table 1. Meropenem levels obtained were $3.5-96.9 \mathrm{mg} / \mathrm{L}$, with all patients obtaining $40 \% f \mathrm{~T}>5 \mathrm{xMIC}$ (free 
Table 1. Attainment of PK targets for meropenem and piperacillin-tazobactam

\begin{tabular}{|c|c|c|c|}
\hline Therapeutic target reached & $\begin{array}{c}50 \% \mathrm{fT}>5 \times \mathrm{MIC}(\mathrm{PT}), 40 \% \mathrm{fT}>5 \times \mathrm{MIC}(\mathrm{M}) \\
\text { n (\%) }\end{array}$ & $\begin{array}{c}100 \% \mathrm{fT}>\mathrm{MIC} \\
\mathrm{n}(\%)\end{array}$ & $\begin{array}{c}100 \% \mathrm{fT}>5 \times \mathrm{MIC} \\
\mathrm{n}(\%)\end{array}$ \\
\hline \multicolumn{4}{|l|}{ Pre-steady state } \\
\hline Meropenem (M) & $17 / 17(100)$ & $17 / 17(100)$ & $12 / 17(70.6)$ \\
\hline Piperacillin-tazobactam (PT) & $4 / 12(33.3)$ & $7 / 12(58.3)$ & $3 / 12(25.0)$ \\
\hline \multicolumn{4}{|l|}{ Steady state } \\
\hline Meropenem (M) & $15 / 16(93.8)$ & $14 / 16(87.5)$ & $5 / 16(31.3)$ \\
\hline Piperacillin-tazobactam (PT) & $4 / 10(40.0)$ & $7 / 10(70.0)$ & $2 / 10(20.0)$ \\
\hline
\end{tabular}

$\% f \mathrm{~T}>\mathrm{MIC}$ : free drug concentrations above bacterial minimum inhibitory concentrations (MIC) over a desired percentage of the dosing period; M: meropenem; PT: piperacillin-tazobactam

Protein binding was assumed to be $2 \%$ for meropenem and $30 \%$ for piperacillin.

For patients without cultures, the most conservative MIC breakpoint of potential pathogens according to the Clinical and Laboratory Standards Institute, US were assumed ( 2 or $4 \mathrm{mg} / \mathrm{L}$ for meropenem and $16 \mathrm{mg} / \mathrm{L}$ for piperacillin).

meropenem concentrations at least 5 times the MIC for $40 \%$ of the dosing interval) within 48 hours, and $15 / 16(93.8 \%)$ at steady state. In contrast, piperacillin levels were $7.3-302.5 \mathrm{mg} / \mathrm{L}$, with only $4 / 12(33.3 \%)$ of patients obtaining $50 \% f \mathrm{~T}>5 \mathrm{xMIC}$ (free piperacillin concentrations at least 5 times the MIC for $50 \%$ of the dosing interval) within 48 hours, and $4 / 10$ $(40.0 \%)$ at steady state. Among the patients with nonsusceptible organisms, the patient with piperacillintazobactam MIC $\geq 128 \mathrm{mg} / \mathrm{L}$ did not achieve any of the PK targets in Table 1. The 30-day all-cause mortality is $5 / 16(31.3 \%)$ for patients on piperacillin-tazobactam and $10 / 26(38.5 \%)$ for patients on meropenem.

This study observed that majority of the critically ill patients in our population achieved the PK targets chosen for meropenem. However, a multinational point prevalence study, the Defining Antibiotic Levels in Intensive care unit patients (DALI) study, described $<70 \%$ of their population achieved $50 \% \mathrm{~T} \mathrm{~T}>4 \mathrm{xMIC}$ or $100 \% \mathrm{~T}>\mathrm{MIC}$ for meropenem. ${ }^{2}$ We postulate that the difference in our findings might be related to differences in the baseline characteristics of the study populations. The smaller build of our Asian population, higher incidence of impaired renal function, lower albumin and age $>60$ compared with the DALI population could have contributed to more patients achieving PK targets for meropenem with standard doses.

Of significant concern, many patients prescribed with piperacillin-tazobactam were not able to achieve the PK targets with the standard doses prescribed.
Discrepant findings between meropenem and piperacillin-tazobactam could be a result of the wide variation in the PK of critically ill patients. This reemphasises the need for strategies such as implementation of a beta-lactam therapeutic drug monitoring service, to improve chances of PK target attainment in our group of patients with extremely unpredictable beta-lactam PK.

This study has notable limitations. Firstly, this is a single-centre study with a relatively small sample size and heterogeneity of infections that may limit generalisability of the results. Secondly, creatinine clearance was calculated using the CockcroftGault formula and was not directly measured. Such calculations generally provide poor estimates at extremes of creatinine clearance or unstable renal function, and may not be optimal for accurate dosing, despite being commonly used clinically. ${ }^{8}$ Thirdly, more than half of our patients did not have a causative pathogen isolated. Therefore, the PK targets and associated results represented the worst-case scenario where the presence of the least susceptible pathogen was assumed. Lastly, the current analysis only provides data on PK exposure and not clinical outcomes. The impact of antibiotics PK target attainment on clinical outcome was not evaluated.

Further studies are needed to define optimal dosing regimens in our Singapore population with diverse PK profiles, and to evaluate the clinical implications and outcomes of using therapeutic drug monitoring to attain PK targets. 


\section{REFERENCES}

1. Guilhaumou R, Benaboud S, Bennis Y, et al. Optimization of the treatment with beta-lactam antibiotics in critically ill patientsguidelines from the French Society of Pharmacology and Therapeutics (Société Française de Pharmacologie et Thérapeutique-SFPT) and the French Society of Anaesthesia and Intensive Care Medicine (Société Française d'Anesthésie et Réanimation-SFAR). Crit Care 2019;23:104.

2. Roberts JA, Paul SK, Akova M, et al. DALI: defining antibiotic levels in intensive care unit patients: are current $\beta$-lactam antibiotic doses sufficient for critically ill patients? Clin Infect Dis 2014; 58:1072-83.

3. Jacobs A, Taccone FS, Roberts JA, et al. $\beta$-lactam dosage regimens in septic patients with augmented renal clearance. Antimicrob Agents Chemother 2018;62:e02534-17.

4. Lo AH, Kee AC, Li A, et al. Controversies in Sepsis ManagementWhat is the Way Forward? Ann Acad Med Singap 2020;49:661-8.

5. Zander J, Döbbeler G, Nagel D, et al. Piperacillin concentration in relation to therapeutic range in critically ill patients--a prospective observational study. Crit Care 2016;20:79.

6. Muller AE, Huttner B, Huttner A. Therapeutic drug monitoring of beta-lactams and other antibiotics in the intensive care unit: which agents, which patients and which infections? Drugs 2018; 78:439-51.

7. Wong G, Brinkman A, Benefield RJ, et al. An international, multicentre survey of $\beta$-lactam antibiotic therapeutic drug monitoring practice in intensive care units. J Antimicrob Chemother 2014;69:1416-23.

8. Sime FB, Udy AA, Roberts JA. Augmented renal clearance in critically ill patients: etiology, definition, and implications for beta-lactam dose optimization. Curr Opin Pharmacol 2015;24:1-6.

Grace Si Ru $\underline{\operatorname{Hoo}}^{1,2}$ MSc (ID), Nathalie Grace $\underline{\text { Chua }}{ }^{3}$ PharmD, Tat Ming $\mathrm{Ng}^{1}{ }_{\text {Pharm }}$, Lawrence Lee ${ }^{4}$ PhD, Andrea Layhoon Kwa ${ }^{3,5,6}$ PharmD, Li Min Ling ${ }^{7}{ }_{M R C P}$

${ }^{1}$ Department of Pharmacy, Tan Tock Seng Hospital, Singapore

${ }^{2}$ Department of Pharmacy, National Centre for Infectious Diseases, Singapore

${ }^{3}$ Department of Pharmacy, Singapore General Hospital, Singapore

${ }^{4}$ Department of Infectious Disease, National Centre for Infectious Diseases, Singapore

${ }^{5}$ Department of Pharmacy, National University of Singapore, Singapore

${ }^{6}$ Emerging Infectious Diseases, Duke-NUS Medical School, Singapore

${ }^{7}$ Rophi Clinic, Gleneagles Medical Centre, Singapore

Correspondence: Dr Li Min Ling, Rophi Clinic, Gleneagles Medical Centre, 6 Napier Road, Suite \#03-13, Singapore 258499.

Email:1mling07@gmail.com 